\title{
SEQUENCES IN GENETIC ALGEBRAS FOR OVERLAPPING GENERATIONS
}

\author{
by I. HEUCH \\ (Received 26th April 1971)
}

\section{Introduction}

When Etherington (2) introduced linear commutative non-associative algebras in connection with problems in theoretical genetics, he pointed out that various sequences of elements in these algebras represented different mating systems. In all such systems it was however assumed that the generations did not overlap, and this restriction has been kept in later work in this field. In this paper we treat sequences which make it possible to find the probability distribution in successive generations in a discrete time model where the generations may be overlapping. We also consider idempotents in genetic algebras and outline how the method used on the overlapping generation sequence may be applied to other sequences.

Unless otherwise stated all algebras considered have the complex numbers as the scalar field. Capital letters are used for all algebra elements.

\section{Classification of algebras considered in genetics}

Etherington (2) defines a baric algebra to be one that admits a non-trivial homomorphism $w$ to the scalar field. $w$ is called the weight function. Let the equation of lowest possible degree connecting the principal powers $G^{j}=G^{j-1} G$ of the general element $G$ in the baric algebra $\mathscr{A}$ be

$$
G^{r}+\theta_{1} G^{r-1}+\ldots+\theta_{r-1} G=0 .
$$

If $\theta_{1}, \theta_{2}, \ldots, \theta_{r-1}$ only depend on the weight $w(G)$ of $G$, then $\mathscr{A}$ is called a train algebra (2). For elements with weight 1 , it is often convenient to factorise (2.1) formally:

$$
G(G-1)\left(G-\lambda_{1}\right) \ldots\left(G-\lambda_{r-2}\right)=0 .
$$

Here $1, \lambda_{1}, \ldots, \lambda_{r-2}$ are the principal train roots.

Our main interest will be in what Gonshor (7) has defined as genetic algebras. $\mathscr{A}$ is a genetic algebra (from now on written GA) if there exists a basis $C_{0}, C_{1}, \ldots, C_{n}$ with multiplication table given by

$$
C_{i} C_{j}=\sum_{k=0}^{n} \gamma_{i j k} C_{k}
$$


where

$$
\begin{aligned}
& \text { (i) } \gamma_{000}=1 \text {; (ii) } \gamma_{0 j k}=0 \text { for } k<j, \quad j=1,2, \ldots, n \text {; } \\
& \text { (iii) } \gamma_{i j k}=0 \text { for } k \leqq \max (i, j), \quad i, j=1,2, \ldots, n \text {. }
\end{aligned}
$$

Then $\mathscr{A}$ is baric, and necessarily $w\left(C_{0}\right)=1, w\left(C_{j}\right)=0$ for $j \geqq 1$. A special train algebra (abbreviated to STA) is a baric algebra where the nilideal consisting of elements with weight 0 is nilpotent, and where all powers of the nilideal are ideals. It follows from the treatment in (4) that a STA is always a $\mathrm{GA}$, and that a GA is a train algebra. Furthermore, according to Theorem 2.1 in (7), the definition of a GA given above is equivalent to the one given by Schafer (13).

A basis in a GA giving a multiplication table of the form (2.3) is called canonical, and the scalars $\gamma_{000}=1, \gamma_{011}, \ldots, \gamma_{0 n n}$ are the train roots. They make up the set of principal train roots (6), perhaps with higher multiplicities, except for $\frac{1}{2}$ that can be a train root without being a principal root. It is easily seen that a change of the first element in a canonical basis will not affect the train roots, and moreover, since the train roots in each case may be regarded as the eigenvalues of the linear transformation consisting of multiplication by this first element, two different bases will always give identical train roots.

Only those linear combinations of the original genetic basis elements which have real (and non-negative) coefficients, can be given any probability interpretation, so it is natural to try to use the real numbers as the scalar field. We would then define real train algebras, real genetic algebras and real special train algebras. But then it is no longer true that a (real) STA is a (real) GA. For any given real STA, the complex algebra with the same multiplication table will however be a GA, and hence a train algebra. From Etherington's treatment (4) it then follows that the train roots in this case will be solutions of equations with real coefficients, and so the train roots with non-zero imaginary part will occur in conjugate pairs. Hence the equation of (2.1) for this algebra will have real coefficients. Since this equation is also satisfied in the original real algebra, every real STA will be a real train algebra.

As an example consider the algebra $\mathscr{A}$ describing the usual one locus case with three alleles $A_{0}, A_{1}, A_{2}$, when during one generation $A_{0}$ certainly mutates to $A_{1}, A_{1}$ to $A_{2}$ and $A_{2}$ to $A_{0}$. The multiplication table is

$$
\begin{array}{r}
A_{0}^{2}=A_{1} ; A_{1}^{2}=A_{2} ; A_{2}^{2}=A_{0} ; A_{0} A_{1}=\frac{1}{2}\left(A_{1}+A_{2}\right) ; A_{0} A_{2}=\frac{1}{2}\left(A_{0}+A_{1}\right) ; \\
A_{1} A_{2}=\frac{1}{2}\left(A_{0}+A_{2}\right) .
\end{array}
$$

With a new basis consisting of

$$
D_{0}=\frac{1}{3}\left(A_{0}+A_{1}+A_{2}\right) ; D_{1}=A_{0}-A_{1} ; D_{2}=A_{1}-A_{2},
$$

the only non-zero products in the multiplication table are

$$
D_{0}^{2}=D_{0} ; D_{0} D_{1}=\frac{1}{2} D_{2} ; D_{0} D_{2}=-\frac{1}{2}\left(D_{1}+D_{2}\right) \text {. }
$$


Thus $\mathscr{A}$ is a real STA, since all powers of the nilideal $\left(D_{1}, D_{2}\right)$ with index greater than 1 contain nothing but zero. To establish that the corresponding complex algebra is a $\mathrm{GA}$, we pass to the basis $C_{0}, C_{1}, C_{2}$ :

$$
\begin{aligned}
& C_{0}=\left(A_{0}+A_{1}+A_{2}\right) / 3 ; \quad C_{1}=A_{0}+e^{-2 \pi i / 3} A_{1}+e^{2 \pi i / 3} A_{2} ; \\
& C_{2}=A_{0}+e^{2 \pi i / 3} A_{1}+e^{-2 \pi i / 3} A_{2} .
\end{aligned}
$$

Then the table can be written

$$
C_{0}^{2}=C_{0} ; C_{0} C_{1}=\frac{1}{2} e^{2 \pi i / 3} C_{1} ; C_{0} C_{2}=\frac{1}{2} e^{-2 \pi i / 3} C_{2},
$$

other products being zero. Every $G$ with $w(G)=1$ satisfies the symbolic equation $G(G-1)\left(G^{2}+\frac{1}{2} G+\frac{1}{4}\right)=0$, which holds in the original real algebra, too. That it is impossible to find any basis transformation giving the form of a real GA, follows from the uniqueness of the train roots in the complex GA.

\section{Idempotents}

Gonshor (6) has shown that there is exactly one non-zero idempotent in a GA when no train root equals $\frac{1}{2}$. The proof is based on induction with respect to the number of basis elements, a method related to the one we will use in sections 5 and 7. Holgate (10) treats a more general case, and makes an assertion equivalent to the following (see equation (25)). Let exactly $t$ of the train roots in a STA be $\frac{1}{2}$, with corresponding elements $C_{i_{1}}, C_{i_{2}}, \ldots, C_{i_{t}}$ in the canonical basis. Then there is a $t$-parameter family of idempotents if no quantity

$$
C_{0}^{2}, C_{i}, C_{i_{k}}, C_{0} C_{i,}-\frac{1}{2} C_{i j}
$$

has any component relatively to $C_{i_{1}}, \ldots, C_{i_{i}}$.

That this statement is not true in general is seen by considering an algebra $\mathscr{A}$ with basis $C_{0}, C_{1}, C_{2}$ and multiplication given by

$$
C_{0}^{2}=C_{0}+C_{1} ; C_{0} C_{1}=\frac{1}{4} C_{1}+C_{2} ; C_{0} C_{2}=\frac{1}{2} C_{2},
$$

other products of the basis elements being zero. $\mathscr{A}$ is a STA, $C_{0}, C_{1}, C_{2}$ is canonical, and the condition above is satisfied with $t=1$ and $i_{1}=2$. If $G=a_{0} C_{0}+a_{1} C_{1}+a_{2} C_{2}$ is idempotent, then

$$
G=G^{2}=a_{0}^{2} C_{0}+\left(a_{0}^{2}+\frac{1}{2} a_{0} a_{1}\right) C_{1}+\left(2 a_{0} a_{1}+a_{0} a_{2}\right) C_{2},
$$

but this implies that $a_{0}=a_{1}=a_{2}=0$. However, in the way suggested by Gonshor (6), it is easily seen that the proposition above will be correct in every GA if the further restriction is made that the $t$ basis elements with train roots $\frac{1}{2}$ should follow $C_{0}$ with no other elements in between.

\section{Trains}

Suppose that $\left\{G^{(j)}\right\}$ is a sequence of elements that can be constructed from any given element $G=G^{(1)}$ in the baric algebra $\mathscr{A}$. Under certain conditions 
this sequence will form a train. This concept was introduced in (2) but, as pointed out in (5), the definition was rather vague, and a more precise formulation does not seem to have appeared later. (Actually, this term should be applied to the rule for constructing the sequence from $G^{(1)}$, rather than the sequence itself.) The original definition may be interpreted as this:

$\left\{G^{(j)}\right\}$ is a train if there exists an equation

$$
G^{(s+1)}+\theta_{1}(w) G^{(s)}+\ldots+\theta_{s}(w) G^{(1)}=0
$$

which is correct for all $G=G^{(1)}$ in $\mathscr{A}$, where $\theta_{1}(w), \ldots, \theta_{s}(w)$ are functions of the weight $w=w(G)$ only.

In order to establish that particular sequences form trains, it has in practice often been considered sufficient that (4.1) should be satisfied with constant $\theta_{1}, \ldots, \theta_{s}$ for all $G$ with $w(G)=1$ :

$$
G^{(s+1)}+\theta_{1} G^{(s)}+\ldots+\theta_{s} G^{(1)}=0
$$

see for instance section 5 of (4), section 3 of (8), and (9). For sequences which may be regarded as powers of $G$, it is easy to show that this condition implies that we have a train according to the definition above, since if we insert $G / w(G)$ in (4.2) for general $G$ and rearrange, we get an equation (4.1) for all $G$ with non-zero weight. A continuity consideration then gives that $G^{(s+1)}=0$ when $w(G)=0$. (An argument of this type is for example used to derive (4.1) for plenary powers in section 4 of (5).)

We assume that (4.2) is the equation of its kind of lowest possible degree, and that for all sequences considered $w(G)=1$ implies that $w\left(G^{(j)}\right)=1$ for all $j$. Then simply by substituting

$$
G^{(j+s)}, G^{(j+s-1)}, \ldots, G^{(j)} \text { for } G^{(s+1)}, G^{(s)}, \ldots, G^{(1)}
$$

in (4.2) we get a homogeneous difference equation for the sequence $\left\{G^{(j)}\right\}$ :

$$
G^{(j+s)}+\theta_{1} G^{(j+s-1)}+\ldots+\theta_{s} G^{(j)}=0
$$

The train equation (4.2) for the sequence may be symbolically factorised as if the terms $G^{(j)}$ were powers of ordinary numbers:

$$
G(G-1)\left(G-\mu_{1}\right) \ldots\left(G-\mu_{s-1}\right)=0 .
$$

( 1 will be a symbolic root in (4.2), as may be seen by taking the image of this equation element by element, under the homomorphism $w$.) When the train roots $1, \mu_{1}, \ldots, \mu_{s-1}$ for the particular sequence are known in addition to the values of $G^{(1)}, G^{(2)}, \ldots, G^{(s)}$, then it is in principle possible to solve (4.3). We find

$$
G^{(j)}=\sum_{u=1}^{t} Q_{u} y_{u}^{j}
$$

where $y_{1}, y_{2}, \ldots, y_{t}$ are the different values of $1, \mu_{1}, \ldots, \mu_{s-1}$, and $Q_{1}, \ldots, Q_{t}$ are elements in $\mathscr{A}$ which are polynomials in $j$.

This solution is valid for all $G$ with weight 1 . However, by using (4.5) for 
$G / w(G)$, we immediately obtain an expression for $G^{(j)}$ when $G$ has any nonzero weight. It should be noticed that in general it is not possible to use (4.1) in a similar way to derive a difference equation corresponding to (4.3). If we substitute $G^{(j+s)}, \ldots, G^{(j)}$ for $G^{(s+1)}, \ldots, G^{(1)}$ in (4.1), an equation is found where the coefficients depend not only on the weight $w=w(G)$ of $G$, but on $j$ too. This difference equation with variable coefficients cannot be solved in the same manner as (4.3). (In the particular case of principal powers, however, the difference equation found from (4.1) has constant coefficients.)

These considerations apply to the case where the sequence is generated by a single element $G=G^{(1)}$. In section 5 we will derive sequences from a number of independent initial elements $(\{H(n)\}$ in (12) also belongs here). The original definition might then be generalised by requiring (4.1) to hold with $\theta_{1}, \ldots, \theta_{s}$ being functions of the various weights of the given elements. This seems however to be too restrictive, and we will give reasons for considering (4.2) instead of (4.1).

Regarding sequences as plenary powers $G^{(j+1)}=G^{(j)} G^{(j)}$, or principal powers, we may interpret as a population any algebra element with non-negative coordinates (and at least one positive) relatively to the original basis elements. The proper probability distribution will in each case be found by assuming that the genotype probabilities should be proportional to the coordinates. Thus it makes sense for the applications in these cases to use $G$ with $w(G) \neq 1$. When we pass to sequences like the one to be defined by (5.1), however, such a use will in general destroy our probability interpretation of the mating system represented by the sequence, and it is essential that all $G$ connected with distributions should be normalised. This sequence will also be different in the following respect. Even when the general expression for $G^{(j)}$ is known for the case where all initial elements have weight 1 , it will be impossible to deduce immediately a similar expression given initial elements with arbitrary weight. The algebraic treatment in the general case would have to be quite different.

Thus it seems reasonable to base our general definition of a train on (4.2), which will be required to hold with constant coefficients for all sets of given elements having weight 1 . Even for the usual sequences of powers, the essential equation is (4.2), not (4.1), since (4.2) gives the difference equation used.

For a train $\left\{G^{(j)}\right\}$ and a particular choice $C_{0}, C_{1}, \ldots, C_{n}$ of canonical basis in a GA, assume that

$$
G^{(j)}=C_{0}+\sum_{i=1}^{n} a_{i j} C_{i}
$$

for elements with weight 1 . We will introduce the concept of a root for the basis element $C_{i}$ (with respect to $\left\{G^{(j)}\right\}$ ). The scalars $r_{i 1}, r_{i 2}, \ldots, r_{i v}$ shall be the roots for $C_{i}$ if each $a_{i j}$ defined by (4.6) may be written

$$
a_{i j}=\sum_{u=1}^{v} q_{i u} r_{i u}^{j}
$$


where the $q_{i u}$ are polynomials in $j$. Then $a_{i j}, j=1,2, \ldots$, satisfies a homogeneous difference equation with characteristic roots $r_{i 1}, \ldots, r_{i v}$. It is understood that the sequence should not satisfy such a difference equation of lower degree. The root $r_{i u}$ has multiplicity $m_{i u}$ if it is a $m_{i u}$-multiple root in the characteristic equation. This amounts to saying that $q_{i u}$ in (4.7) is a polynomial in $j$ of degree $m_{i u}-1$. This rule must be modified for a root equal to 0 . If 0 has multiplicity $m$ for $C_{i}$, then (4.7) is valid only for $j \geqq m+1, r_{i 1}, \ldots, r_{i v}$ now being the nonzero roots. The multiplicity of a root in the complete algebra is defined similarly.

\section{The sequence for overlapping generations}

For given algebra elements $G^{(1)}, G^{(2)}, \ldots, G^{(p)}$ this sequence is defined by

$$
G^{(j+p)}=\sum_{k=0}^{p-1} \sum_{h=0}^{p-1} b_{h k} G^{(j+h)} G^{(j+k)}, j=1,2, \ldots,
$$

where $p$ is a given natural number, and $b_{h k}, h, k=0,1, \ldots, p-1$, are given scalars such that $b_{h k}=b_{k h}, \sum_{h, k} b_{h k}=1$, and $b_{0 k} \neq 0$ for at least one $k$. If $w\left(G^{(1)}\right)=\ldots=w\left(G^{(p)}\right)=1$, then automatically $w\left(G^{(j)}\right)=1$ for all $j$. We introduce the notation $b_{h} .=\sum_{k} b_{h k}$. Concerning the algebra $\mathscr{A}$, we now suppose that it is a GA with canonical basis $C_{0}, C_{1}, \ldots, C_{n}$, satisfying (2.2) and (2.3). The train roots $\gamma_{0 j j}$ will be written as $\lambda_{j}$. Then we have

Theorem 5.1. (i) The sequence $\left\{G^{(j)}\right\}$ defined by (5.1) is a train in $\mathscr{A}$.

(ii) $C_{0}$ has the one and only root 1 , with multiplicity 1.

(iii) For $i \geqq 1$ we have: The roots for $C_{i}$ are elements of the union of the set of products of all pairs of roots for $C_{t}$ and $C_{s}$ with $0 \leqq t, s<i$ and $\gamma_{t s i} \neq 0$, and the set of solutions of the equation

$$
x^{p}-2 \lambda_{i} b_{p-1} \cdot x^{p-1}-\ldots-2 \lambda_{i} b_{0 .}=0 .
$$

(iv) The multiplicity of a root $(\neq 0)$ formed by such a product is $\leqq$ (the sum of the multiplicities for each root) -1 . When more than one product give the same root, the relevant multiplicity is $\leqq$ (maximum of the upper bounds calculated in the described manner). The multiplicity of a root found from (5.2) is equal to the multiplicity as a solution of this equation. If a root is found in both ways, we get an upper bound by adding the two bounds. A product equal to 0 will give a multiplicity equal to maximum mult iplicity of 0 for the factor roots.

(v) The set of roots for $\mathscr{A}$ is the union of all sets of roots for the elements in the basis. The multiplicity is given by the maximum multiplicity for this root for $C_{0}, C_{1}, \ldots, C_{n}$.

Proof. We consider arbitrary $G^{(1)}, \ldots, G^{(p)}$ all having weight 1 . Then (ii) is trivially correct. We will now prove by induction with respect to $i$ that every 
$a_{i j}$ may be written as in (4.7) (the $q_{i u}$ being determined by $a_{i 1}, \ldots, a_{i p}$ ), and that the quantities $r_{i u}$ are given by the rules in (iii) and (iv). Assume this to be true for $i=g-1$. When we insert (4.6) in (5.1) and collect the coefficients of $C_{g}$, using the properties of the GA, we find

$$
a_{g, j+p}-2 \lambda_{g} \sum_{k=0}^{p-1} b_{k} \cdot a_{g, j+k}=\sum_{t=0}^{g-1} \sum_{s=0}^{g-1} \gamma_{t s g} \sum_{h=0}^{p-1} \sum_{k=0}^{p-1} b_{h k} a_{t, j+h} a_{s, j+k}
$$

Only $C_{t}$ and $C_{s}$ with $\gamma_{t s g} \neq 0$ give actual terms on the right-hand side of (5.3). According to the induction hypothesis we may insert expressions (4.7) for $a_{t, j+h}$ and $a_{s, j+k}$, and after rearrangement the right-hand side of (5.3) may be written

$$
\sum_{t, s} \sum_{u, w} c_{t s u w}\left(r_{t u} r_{s w}\right)^{j}
$$

where the first sum is to be taken over $t$ and $s$ within the limits $0 \leqq t, s \leqq g-1$ which give $\gamma_{t s g} \neq 0$. $\quad c_{t s u w}$ is a polynomial in $j$ of degree $\left(m_{t u}-1\right)+\left(m_{s w}-1\right)$. Now (5.3) may be regarded as a usual inhomogeneous difference equation for the sequence $a_{g j}, j=1,2, \ldots$, with known right-hand side (5.4), and the corresponding homogeneous equation will have characteristic equation (5.2) (with $i=g$ ). (5.3) may then be solved in the ordinary way (see for instance section 171 of (11)), giving an expression (4.7) for $a_{g j}$, with $r_{g u}$ constructed in one or both of the two manners described in (iii). The sequence $a_{g j}$ will also satisfy a homogeneous difference equation where the characteristic equation has all $r_{g u}$ as solutions. If $r_{g u}$ is only formed as a product $r_{t u} r_{s w}$, the degree of the polynomial $q_{g u}$ will be $m_{t u}+m_{s w}-2$. The characteristic equation just mentioned must then in general have $r_{g u}$ as $\left(m_{t u}+m_{s w}-1\right)$-multiple root. If $r_{g u}$ is only obtained from (5.2) with multiplicity $m^{\prime}$ then $q_{g u}$ is of degree $m^{\prime}-1$, and $r_{g u}$ will have to be a solution of multiplicity $m^{\prime}$ of the characteristic equation. It is also seen that the boundaries may be added when $r_{g u}$ is found in both ways. This completes the induction.

The solutions $a_{i j}$ for the basis elements may now be put together to a solution for the complete $G^{(j)}$. We find that $G^{(j)}$ will satisfy a homogeneous difference equation with all $r_{i u}$ as characteristic roots, the multiplicities being at least as great as the corresponding multiplicities for each basis element. This proves (i) and (v).

In particular cases some of the coefficients in the polynomials $c_{\text {tsuw }}$ in (5.4) may turn out to be 0 . For this reason the rules above for multiplicities only give upper bounds. If we had regarded all roots with multiplicity $m>1$ as if we had $m$ different roots, used (iii) instead of (iv), and in the end estimated the multiplicity of a root by counting the number of times it occurred, we would find greater upper bounds than those obtained from (iv); when we ought to have $m_{1}+m_{2}-1$, we would find $m_{1} m_{2}$.

Now assume that $\mathscr{A}$ is a GA defined over the real numbers instead of the complex ones, and that all $b_{h k}$ are real. If (5.2) still has complex solutions, then 
these must appear in conjugate pairs. When we now pass to the corresponding complex algebra, as a consequence of (iii) we find that all roots with non-zero imaginary part will be given in such pairs. Furthermore, because of symmetry, when one root vanishes in (5.4) so does its conjugate, and the two will always have the same multiplicity. The homogeneous difference equation equivalent to (5.3) of lowest possible degree can accordingly be written with only real coefficients, and this will also be the case for the train equation, which is satisfied by every $G$ in the original real GA. Thus the theorem is valid for real algebras, too. Difficulties will arise, though, when we write down the expression (4.5). But here we " get rid of " the imaginary parts as usual: If $y_{u}=y=\rho e^{i \phi}$, we may substitute $R \rho^{j} \cos (j \phi)+R^{\prime} \rho^{j} \sin (j \phi)$ in (4.5) for $Q y^{j}+Q^{\prime} \bar{y}^{j}$, where $R$ and $R^{\prime}$ are elements in the real algebra, being polynomials in $j$ of the same degree as $Q$ and $Q^{\prime}$.

It may be noticed that for initial elements $G^{(1)}, \ldots, G^{(p)}$ all having weight 0 , $G^{(j)}=0$ for all sufficiently large $j$, since every product in the nilideal (in a GA) whose degree exceeds a certain value will be 0 .

Theorem 5.1 is easily extended to more general sequences. Almost the same arguments apply to

$$
G^{(j+p)}=\sum_{h, k} b_{h k}\left(G^{(j+h)} G^{(j+k)}\right)+\sum_{k=0}^{p-1} d_{k}\left(P_{k} G^{(j+k)}\right),
$$

when $b_{h k}$ and $d_{k}$ are given scalars with $\sum_{h, k} b_{h k}+\sum_{k} d_{k}=1$, and $P_{0}, P_{1}, \ldots, P_{p-1}$ are given algebra elements with weight 1 . For each pair $C_{t}, C_{s}$ with $\gamma_{t s i} \neq 0$, the set of roots for $C_{i}$ will then in addition contain the roots of $C_{t}$ if any $P_{k}$ has non-zero coefficient relatively to $C_{s}$, and under the corresponding conditions the roots of $C_{s}$. The rules for multiplicity are easily modified, and (5.2) is replaced by

$$
x^{p}-\lambda_{i}\left(2 b_{p-1} .+d_{p-1}\right) x^{p-1}-\ldots-\lambda_{i}\left(2 b_{0} .+d_{0}\right)=0 .
$$

We set $b=\Sigma b_{h k}$ and $d=\Sigma d_{k}$. When all $b_{h k}$ and $d_{k}$ are real and non-negative and all $\left|\lambda_{i}\right| \leqq 1 /(2 b+d)$, then (5.6) can have no solutions outside the unit circle, because if $|x|>1$, then (5.6) would imply

$$
|x|^{p} \leqq\left|\lambda_{i}\right| \sum_{k=0}^{p-1}\left(2 b_{k}+d_{k}\right)|x|^{k} \leqq|x|^{p-1} .
$$

If $\left|\lambda_{i}\right|<1 /(2 b+d)$, there can be no solutions on the unit circle either, and it then follows that if 1 is a root for any $C_{i}$, it must have multiplicity 1 . All other roots will have modulus less than 1 , accordingly $\lim _{j \rightarrow \infty} a_{i j}$ will exist, and so will $\lim G^{(j)}$. With $P=\sum_{k} d_{k} P_{k} / d$, it now follows from (5.5) that the limit $L$ for $G^{(j)}$ will satisfy an equation

$$
L=b L^{2}+d P L
$$


which is easily shown to have exactly one non-zero solution in this case. (For $d=0$ the last term in (5.7) is omitted. Thus sequences (5.1) will have idempotent limits.) These results may be considered as generalisations of Theorem 2.2 in (6).

\section{Applications}

The sequence (5.1) may be applied to an infinite population where mating takes place only at given points of time, but where the generations can overlap. We suppose that the individuals participate in mating for the last time when they reach an age of $p$ generations. Each time a proportion $2 b_{h k}$ of the crosses are made between individuals of age $p-h$ and $p-k$ (for $h \neq k ; b_{h k}$ if $h=k$ ). Apart from this restriction mating is assumed to be random. $G^{(j)}$ will now give the distribution of genotypes among individuals born at time $j$, when the distributions among the initial $p$ generations are known, that is when $G^{(1)}, \ldots, G^{(p)}$ are given.

For particular choices of $b_{h k},(5.1)$ will reduce to cases studied before. With $p=1, b_{00}=1$ we get the sequence of plenary powers. The sequence

$$
H(n)=H(n-1) H(n-2)
$$

introduced by Reiersøl (12) in connection with sex-linked loci is obtained from (5.1) with $p=1, b_{01}=b_{10}=\frac{1}{2}$. Some other special cases of (5.1) have also been treated by Reiersøl by means of differential operators (unpublished).

Using (5.5), one may imagine that in addition to the crosses between individuals of different age, a proportion $d_{k}$ of the crosses at time $j$ are made between individuals in our sequence of age $p-k$ and a given constant population with distribution determined by $P_{k}$. With $p=1, b_{00}=0, d_{0}=1$ and $P_{0}=G^{(1)}$ we here get the ordinary sequence of principal powers. Thus we have a new proof for the fact that every GA is a train algebra.

It may be of interest to compare Theorem 5.1 used on plenary powers with previous results in the same direction. For this sequence $x=2 \lambda_{i}$ is the only solution of (5.2). Etherington has shown in (3) that in every train algebra of rank 3 with train roots 1 and $\lambda$, the plenary powers will form a train with roots 1 and $2 \lambda$. Holgate's Theorem 3 in (9) is more relevant. It is seen that our Theorem 5.1 applied to this sequence is more general than that theorem in these ways: (i) We consider a GA instead of a STA; (ii) it is no condition that the algebra must contain an idempotent; (iii) we drop products formed from $C_{t}, C_{s}$ with $\gamma_{t s i}=0$; and (iv) we give smaller bounds for the multiplicities. For particular applications, especially (iii) is important. Starting with $G=\sum_{i=0}^{n} u_{i} C_{i}$ the proof in (9) introduces new quantities $v_{1}, \ldots, v_{m}$ making squaring of $G$ equivalent to a linear transformation on $v_{1}, \ldots, v_{m}$. It is proved by induction that $v_{1}, \ldots, v_{m}$ of this kind can be found, but the deductions corresponding to our solution 
of the difference equations and the construction of the roots are made simultaneously for the coordinates relatively to all elements in the basis. Thus one might say that the proof of Theorem 3 in (9) does not fully utilise the preceding Theorem 1. The equivalent of this Theorem 1 has been used in setting up our equation (5.3).

\section{A general sequence of powers}

The same technique of induction with regard to the number of elements in the basis may be applied to many other sequences in a GA, and so these will form trains. As an example consider $\left\{G^{(j)}\right\}$ defined by

$$
G^{(j+1)}=\left(G^{(j)}\right)^{s}, \quad j=1,2, \ldots,
$$

where $\left(G^{(j)}\right)^{s}$ stands for any non-associative power of $G^{(j)}$, characterised by its (commutative) shape $s$ (1). An arbitrary factor element in the product corresponding to $s$ will be denoted by $f$. The shape $s$ may be visualised as a tree (or a pedigree) as described in (1) and then each such element $f$ is represented by a terminal node in the tree. The distance between this node and the node $f_{0}$ representing the final product, measured in generations (or the number of edges along the path connecting $f$ and $f_{0}$ ), will be written as $h(f)$. The maximum value of $h(f)$ for all $f$ is the altitude $\alpha(s)$ of the shape (1). For instance

$$
h(X)=h(Y)=3, h(Z)=2, h(V)=1
$$

in the primary shape $s=4$ given by the product $((X Y) Z) V$.

The same notation (4.6) is used as before. We first establish a difference equation similar to (5.3). Using distributivity, we see that $\left(G^{(j)}\right)^{s}$ may be expressed as a linear combination of a number of products all having shape $s$, where the factors consist exclusively of basis elements $C_{0}, C_{1}, \ldots, C_{n}$. Imagine that all multiplications are performed and that each product is written out as a linear combination of the basis elements. Then, because of (2.3), only those products where all factor elements except one were equal to $C_{0}$, and the remaining one, say $f$, was $C_{g}$, will give any term $a_{g j} C_{g}$ multiplied by a scalar (not depending on any $a_{i j}$ ). For products of this kind we get the term $\lambda_{g}^{h(f)} a_{g j} C_{\theta}$. Other components relatively to $C_{g}$ are obtained, but these will be of the form $a_{i_{1}, j} \ldots a_{i_{v}, j} C_{g}$ multiplied by scalars, where all $i_{t}$ are less than $g$. Hence, equating the coefficient of $C_{g}$ in this expression with $a_{g, j+1}$ we get a difference equation with left-hand side

$$
a_{g, j+1}-\left(\sum_{f} \lambda_{g}^{h(f)}\right) a_{g, j}
$$

and a right-hand side depending on the values of $a_{i j}$ for $i<g$.

Now the same method of solving these equations inductively as the one used for (5.3) may be applied. The roots for $C_{i}$ are found as certain products of roots for basis elements with lower indices, and $\sum_{f} \lambda_{i}^{(f)}$. When all roots are known, the train equation for $\left\{G^{(j)}\right\}$ may be constructed. 
One may compare this with Etherington's result in (3) for the sequences $G^{(j+1)}=\left(G^{(j)}\right)^{3}$ and $G^{(j+1)}=\left(G^{(j)}\right)^{4}$ in the case of arbitrary train algebras of rank 3. The train roots are then given as $1, \lambda+2 \lambda^{2}$ and $1, \lambda+\lambda^{2}+2 \lambda^{3}$, respectively. In these instances $\sum_{f} \lambda_{i}^{h(f)}$ will be $\lambda_{i}+2 \lambda_{i}^{2}$ and $\lambda_{i}+\lambda_{i}^{2}+2 \lambda_{i}^{3}$.

\section{REFERENCES}

(1) I. M. H. Etherington, On non-associative combinations, Proc. Roy. Soc. Edinburgh 59 (1939), 153-162.

(2) I. M. H. Etherington, Genetic algebras, Proc. Roy. Soc. Edinburgh 59 (1939), 242-258.

(3) I. M. H. Etherington, Commutative train algebras of ranks 2 and 3, $J$. London Math. Soc. 15 (1940), 136-149.

(4) I. M. H. Etherington, Special train algebras, Quart. J. Math. Oxford Ser. (2) 12 (1941), 1-8.

(5) I. M. H. Etherington, Non-commutative train algebras of ranks 2 and 3, Proc. London Math. Soc. (2) 52 (1950), 241-252.

(6) H. Gonshor, Special train algebras arising in genetics, Proc. Edinburgh Math. Soc. (2) 12 (1960), 41-53.

(7) H. Gonshor, Contributions to genetic algebras, Proc. Edinburgh Math. Soc. (2) 17 (1971), 289-298.

(8) P. Holgate, Genetic algebras associated with polyploidy, Proc. Edinburgh Math. Soc. (2) 15 (1966), 1-9.

(9) P. Holgate, Sequences of powers in genetic algebras, J. London Math. Soc. 42 (1967), 489-496.

(10) P. Holgate, The genetic algebra of $k$ linked loci, Proc. London Math. Soc. (3) 18 (1968), 315-327.

(11) C. JoRdan, Calculus of Finite Differences, 3rd edition (Chelsea, New York, 1965).

(12) O. ReIRRsøL, Genetic algebras studied recursively and by means of differential operators, Math. Scand. 10 (1962), 25-44.

(13) R. D. SCHAFER, Structure of genetic algebras, Amer. J. Math. 71 (1949), $121-135$.

INSTITUTE OF GENERAL GENETICS

UNIVERSITY OF OSLO

NORWAY 